\title{
Interleukin-6 levels in the serum and saliva of patients with oral lichen planus compared with healthy controls: a meta-analysis study
}

\author{
HAMID REZA MOZAFFARI ${ }^{1}$, ROOHOLLAH SHARIFI ${ }^{2}$, MASOUD SADEGHI $I^{3,4}$ \\ ${ }^{1}$ Department of Oral Medicine, School of Dentistry, Kermanshah University of Medical Sciences, Kermanshah, Iran \\ ${ }^{2}$ Department of Endodontics, School of Dentistry, Kermanshah University of Medical Sciences, Kermanshah, Iran \\ ${ }^{3}$ Medical Biology Research Center, Kermanshah University of Medical Sciences, Kermanshah, Iran \\ ${ }^{4}$ Students Research Committee, Kermanshah University of Medical Sciences, Kermanshah, Iran
}

\begin{abstract}
Interleukin-6 (IL-6) is a cytokine that contributes to the pathogenesis of oral lichen planus (OLP). The aim of this meta-analysis study is the evaluation of IL-6 levels in the serum and saliva of patients with OLP compared with healthy controls. We searched the studies in 5 databases: PubMed/Medline, Scopus, ScienceDirect, Web of Science, and Cochrane Library, from 1983 to Oct 31, 2016. Eleven studies were analysed for the meta-analysis study. The reviewers independently evaluated the quality of each included study using the Newcastle-Ottawa Quality Assessment Scale (NOS). A random-effects meta-analysis, using Comprehensive Meta-Analysis software version 2.0, was used to reflect the variation in studies. Heterogeneity between estimates was evaluated by the $Q$ and $I^{2}$ statistics and for the $Q$ statistic; heterogeneity was considered for $p<0.1$. Eleven studies included 529 OLP patients and 333 healthy controls. The review identified two different biomaterials used for IL-6 assays: saliva and serum. The mean quality score of eleven studies was 7 (high quality). Estimates pooled from 6 studies showed significant high saliva IL-6 levels in OLP patients compared with healthy controls (the standardised difference in means $(S D M)=4.534,95 \% C I=1.915-7.153, p=0.001)$. Also, estimates pooled from 7 studies showed significantly high serum IL-6 levels in OLP patients compared with healthy controls $(S D M=1.482$, 95\% CI =0.524-2.439, $p=0.002$ ). The higher levels of IL-6 in saliva compared with serum suggest that measurement of this marker in saliva may be more useful than serum for diagnostic and therapeutic aims.
\end{abstract}

Key words: lichen planus, interleukin-6, serum, saliva.

(Centr Eur J Immunol 2018; 43 (1): 103-108)

\section{Introduction}

Oral lichen planus (OLP) is a chronic inflammatory and autoimmune disease that affects about $1.9 \%$ [1] to $4 \%$ [2] of the population. It occurs more frequently in females [1, 2], and the immunological system is believed to play a significant role in it [3]. This autoimmune disorder is mediated by $\mathrm{T}$ cells [4] that locally present in the involved tissue release cytokines like interleukin-6 (IL-6), which contributes to the pathogenesis of OLP. IL-6 has been associated with multidrug resistance protein (MRP) expression by keratinocytes [5] that up-regulate MRP expression seen in OLP lesions [6]. Immunological other activities of IL-6 include B-cell differentiation and stimulation of immunoglobulin $\mathrm{G}$ secretion, $\mathrm{T}$ cell differentiation and growth, and cytotoxic $\mathrm{T}$ cell differentiation [7]. The levels of IL-6 increase in the serum of patients with malignant lesions [8]. This interleukin inactivates the p53 tumour suppressor gene [9], promotes tumour cell proliferation [10], and increases the development and progression of some cancers [11], such as oral squamous cell carcinoma [12]. Therefore, levels of IL-6 in the serum and saliva of OLP patients are considered as reliable indicators of therapeutic response on a molecular basis $[2,5,13-21]$. The purpose of this meta-analysis study is to evaluate IL-6 levels in the serum and saliva of patients with OLP compared with healthy controls.

\section{Material and methods}

\section{Search strategies and study criteria}

We searched the studies in 5 databases (PubMed/Medline, Web of Science, ScienceDirect, Cochrane Library, and Scopus) from 1983 to Oct 31, 2016, for English-language publications, using the keywords "oral lichen planus", "OLP", "interleukin-6", and "IL-6".

Correspondence: Masoud Sadeghi, MSc, Medical Biology Research Center, Kermanshah University of Medical Sciences,

67145-1673 Kermanshah, Iran, e-mail: sadeghi_mbrc@yahoo.com

Submitted: 07.01.2017; Accepted: 20.02.2017 


\section{Study selection}

One reviewer (M.S.) searched the articles, and then the second reviewer (H.R.M.) did the same, blinded to the first reviewer. If there was any disagreement between the two reviewers, a third reviewer (R.S.) resolved the problem. All studies were searched for the evaluation of IL-6 levels in the serum and saliva of patients with OLP compared with healthy controls. Studies were included if: a) they were just case-control studies in the English abstract; b) they assessed IL-6 levels in the serum or saliva of OLP patients; c) they diagnosed OLP based on clinical methods, histological methods, or both by the World Health Organisation (WHO) [21]; d) the control subjects did not have OLP or any other cutaneous dermatological or systematic disease; and e) the OLP patients had no any other dermatological and systematic disease.

\section{Data extraction}

The relevant data extracted from each study included: the name of the author, year of publication, country of origin, number of OLP patients, number of control subjects, male/female ratios for both groups, age for both groups (mean $\pm \mathrm{SD}$ ), and levels of IL-6 (mean \pm SD). IL-6 levels were measured from serum and saliva with the immunoenzymatic technique (ELISA) performed with a human IL-6 high-sensitivity ELISA kit, and different units of measurement were used across the studies (pg/ml or $\mathrm{ng} / \mathrm{l})$. If the data were presented using the standard error $(S E)$, then the formula:

$\mathrm{SE}=\frac{\mathrm{SD}}{\sqrt{\mathrm{N}}}$ was used to calculate $S D(N=$ sample size $)$.

If the data were presented using several SDs, the mean SD was calculated by the formula:

$\mathrm{SD}_{\text {mean }}=\sqrt{\mathrm{SD}_{\text {mean }}^{2}}=\sqrt{\frac{\mathrm{SD}_{A}^{2}}{N_{A}}+\frac{\mathrm{SD}_{B}^{2}}{N_{B}}}+\ldots(N=$ sample size $)$

\section{Quality evaluation}

Two authors (M.S. and H.R.M.) independently evaluated the quality of each included study using the Newcastle-Ottawa Quality Assessment Scale (NOS) to determine the quality of selection, comparability, exposure, and outcome of study individuals, with a maximum score of 9 points. The studies quality was divided into three categories: (I) high quality (scored 7 to 9); (II) moderate quality (scored 4 to 6); and (III) low quality (scored 0 to 3 ). Disagreements were resolved through mutual discussion [22].

\section{Statistical analysis}

A random-effects meta-analysis, using Comprehensive Meta-Analysis software version 2.0 (CMA 2.0) was used to reflect variations in studies. The standardised difference in means (SDM) of the studies were calculated in comparison to the estimate of IL-6 levels in the serum or saliva of patients with OLP compared with control subjects by using meta-analysis. Heterogeneity between estimates was evaluated by the $\mathrm{Q}$ and $\mathrm{I}^{2}$ statistics and for the $\mathrm{Q}$ statistic; heterogeneity was considered for $p<0.1$. Confidence interval (CI) was $95 \%$ and a two-sided $p$ value $<0.05$ was considered to be statistically significant in this study. Publication bias was evaluated through funnel plot analysis with Begg's and Egger's tests.

\section{Results}

Out of 369 studies searched in databases, 57 studies were evaluated for eligibility. Of those 57 studies, 26 studies reported other interleukins (except for IL-6), 12 reported IL-6 in other diseases (except for OLP), two were review studies, two had no healthy controls, two were conference papers, one study reported both LP and OLP as patient groups, and one study had no mean $\pm \mathrm{SD}$, which meant that these studies were excluded from our analysis (Fig. 1). Therefore, 11 studies were analysed for meta-analysis study (Table 2).

\section{Study characteristics}

Table 1 shows the characteristics of 11 studies included in meta-analysis. All studies were published between 1994 and 2015. Four studies were reported in the USA [2, 13, 14, 19], two studies in Taiwan $[15,16]$, two studies in China $[17,18]$, one in Belgium [20], one in Japan [21], and one study in India [9]. These 11 studies included 529 OLP patients (range, 10 to 158 patients) and 333 healthy controls (range, 13 to 77 controls). The mean age of the healthy controls varied from 34.9 [21] to 62.5 years [2]; four studies $[9,13,14,20]$ did not report the mean age. The mean age of the OLP patients varied from 45 [18] to 64.1 years [2]; one study [20] did not report the mean age. The review identified two different bio-materials used for IL- 6 assays: saliva $[13,14,17-20]$ and serum $[2,5,15,16,18,20,21]$. The study by Gu, 2004 [2] had no relevant IL-6 levels of ulcerative OLP patients (two cases had very high IL-6 levels) and therefore we compared the mean and SD of reticular OLP patients with controls.

\section{Meta-analysis of IL-6 levels of OLP patients}

Estimates pooled from 6 studies showed significantly high levels of IL-6 of saliva in OLP patients compared with healthy controls (SDM $=4.534,95 \%$ CI: 1.915-7.153, $p=0.001)$. However, statistically significant heterogeneity was found across the studies $\left(\mathrm{I}^{2}=97.87 \%, p<0.001\right)$ (Fig. 2).

Estimates pooled from seven studies showed a significantly high level of IL- 6 of serum in OLP patients compared with healthy controls $(\mathrm{SDM}=1.482$, 95\% CI: $0.524-2.439, p=0.002)$. However, statistically significant heterogeneity was found across the studies $\left(\mathrm{I}^{2}=96 \%, p<0.001\right)$ (Fig. 3). 


\section{Quality evaluation}

Table 2 shows the quality score for each study in the meta-analysis. The mean quality score of the eleven studies was 7 (high quality).

\section{Publication bias}

Figure 4 shows the symmetric funnel plot. Begg's and Egger's tests did not reveal significant evidence of publication bias among the included studies on saliva (Begg's test, $p=0.188$; Egger's test, $p=0.116$ ) (Fig. 4A). Also, Begg's and Egger's tests did not reveal significant evidence of publication bias among the included studies on serum (Begg's test, $p=0.176$; Egger's test, $p=0.153$ ) (Fig. 4B).

\section{Discussion}

This meta-analysis study indicated six studies on saliva (144 OLP patients and 162 healthy controls) and seven studies on serum (469 OLP patients and 251 healthy controls). A random-effect model was done to calculate the pooled estimates because there was a significant heterogeneity between studies. The pooled SDM showed that IL-6 levels in saliva and serum of OLP patients were significantly higher than healthy controls, whereas IL-6 levels in saliva were higher (around three-fold) than serum. The results of Begg's and Egger's tests also showed a lack of significant publication bias. Ten of the eleven studies in this meta-analysis reported that the IL-6 levels in serum or saliva of OLP patients were higher than healthy controls $(p<0.05)$ (except for Liu's study [17]).

IL-6, a cytokine, increases at the sites of inflammation in autoimmune diseases such as OLP [15]. This cytokine can serve as a biomarker to predict disease severity and monitor disease activity [23]. Kaur et al. [20] checked IL-6 levels of saliva and serum in 54 OLP patients and 50 healthy age-matched controls with smoking and/or alcohol consumption. The results showed no significant difference between both groups for IL-6 levels. Rhodus et al. [19] compared 13 OLP patients (77\% smoker) with 13 age and sexmatched healthy controls (23.1\% smokers) and showed that the levels of this cytokine between smoking and non-smoking groups did not show a significant difference, but in a study of cervical cancer, it was noted that IL-6 levels were higher in the cervical mucus of smokers compared with non-smokers [24]. Another study, on leukoplakia and oral cancer patients [25], reported that smoking did not affect concentrations of IL-6 of serum or saliva, and there was no significant difference in salivary concentration of this cytokine between smokers and non-smokers. Although smoking can be a risk factor of OLP, the results showed that this factor cannot significantly increase IL-6 levels in OLP disease. In a study by Kaur et al. [20] the IL-6 levels of serum and saliva were significantly higher in advanced stages of OLP lesions as compared to early stages. Sun et al. [15] studied 158 patients (27.2\%

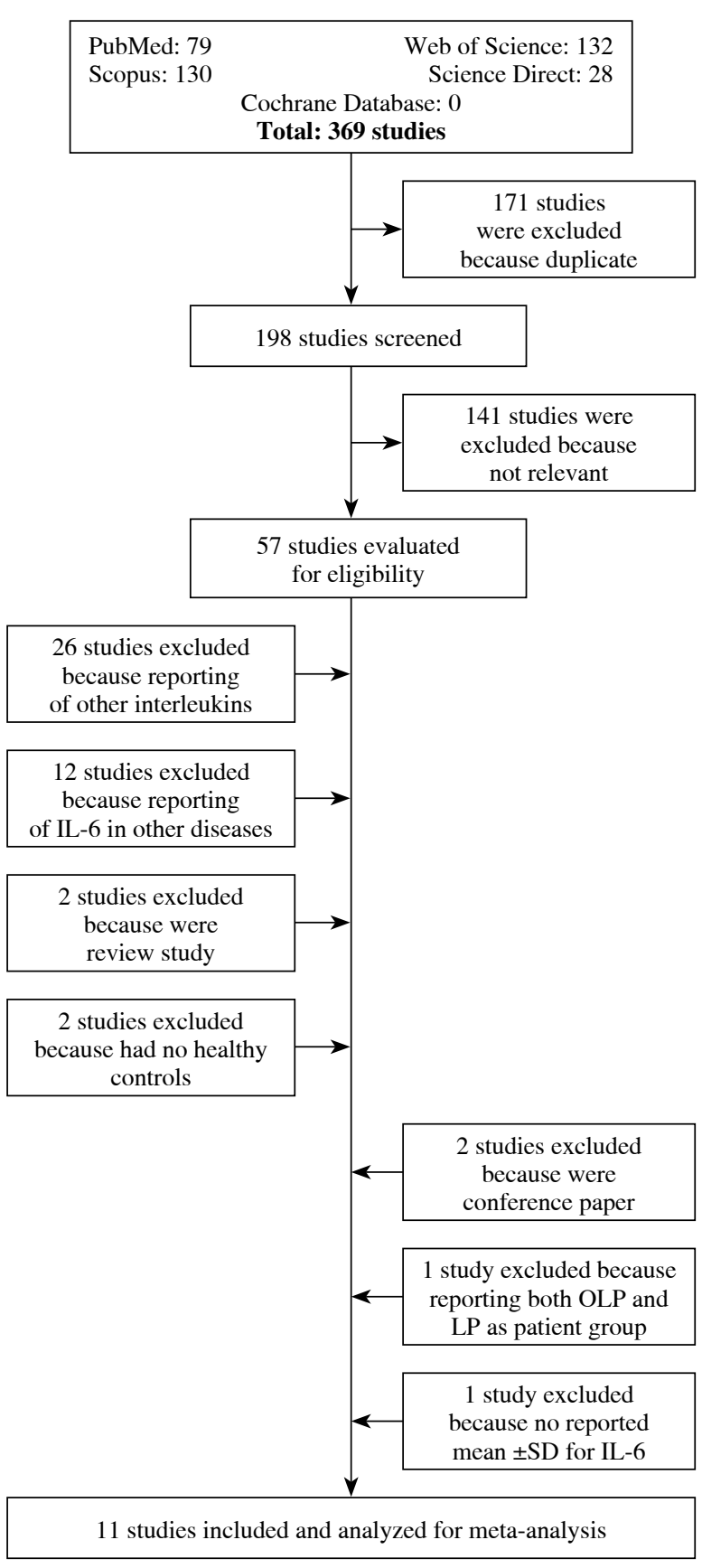

Fig. 1. Flow chart of the study selection processes to identify studies eligible for the systematic review

men, age range 19-79 and mean 51.3 years) with OLP; none of them had taken any prescription medication for around three months before entering the study. The mean level of IL-6 was 5.1 in erosive OLP patients and 4.5 in non-erosive OLP. Another study by Sun et al. [16] checked 149 patients (mean age 53 years, range 19-87 years) with OLP (136 erosive OLP and 13 non-erosive OLP patients); all the patients had not taken any prescription medication at least 
Table 1. The characteristics of the eleven studies included in meta-analysis

\begin{tabular}{lccccccc}
\hline Study & Country & $\begin{array}{c}\text { Number } \\
\text { of controls }\end{array}$ & $\begin{array}{c}\text { Number } \\
\text { of OLP } \\
\text { patients }\end{array}$ & $\begin{array}{c}\text { Sex } \\
\text { of controls, } \\
\mathbf{M ~}: \mathbf{F}\end{array}$ & $\begin{array}{c}\text { Sex of OLP } \\
\text { patients, } \\
\mathbf{M}: \mathbf{F}\end{array}$ & $\begin{array}{c}\text { Mean age } \\
\text { of controls }\end{array}$ & $\begin{array}{c}\text { Mean age } \\
\text { of OLP } \\
\text { patients }\end{array}$ \\
\hline Gu, 2004 [2] & USA & 10 & 10 & $3: 7$ & $1: 9$ & 62.5 & 64.1 \\
\hline Goel, 2015 [5] & India & 10 & 42 & Matched & $8: 7$ & Matched & 46.9 \\
\hline Rhodus, 2005 [13] & USA & 13 & 13 & $0: 13$ & $0: 13$ & 58 & 57 \\
\hline Rhodus, 2006 [14] & USA & 13 & 13 & Matched & $0: 13$ & Matched & 57.2 \\
\hline Sun, 2005 [15] & Taiwan & 54 & 158 & $4: 5$ & $43: 115$ & NR & 51.3 \\
\hline Sun, 2002 [16] & Taiwan & 77 & 149 & $32: 45$ & $46: 103$ & 45 & 53 \\
\hline Liu, 2011 [17] & China & 43 & 21 & $4: 17$ & $15: 28$ & 60.4 & 57.3 \\
\hline Zhang, 2008 [18] & China & 30 & 30 & $1: 1$ & $8: 7$ & 40 & 45 \\
\hline Rhodus, 2005 [19] & USA & 13 & 13 & $3: 10$ & $4: 9$ & 58 & 57 \\
\hline Kaur, 2015 [20] & Belgium & 50 & 54 & - & - & Matched & Matched \\
\hline Yamamoto, 1994 [21] & Japan & 20 & 26 & $1: 1$ & $3: 10$ & 34.9 & 61.2 \\
\hline NR
\end{tabular}

NR-not reported, $M: F$ - male to female, OLP-oral lichen planus

Table 2. Quality assessment of the included studies based on the Newcastle-Ottawa Scale

\begin{tabular}{|c|c|c|c|c|}
\hline Study & Selection & Comparability & Exposure/Outcome & Total score \\
\hline $\mathrm{Gu}, 2004$ [2] & $* * * *$ & $* *$ & $* *$ & 8 \\
\hline Goel, 2015 [5] & $* * *$ & $* *$ & $* *$ & 7 \\
\hline Rhodus, 2005 [13] & $* * *$ & $* *$ & $* *$ & 7 \\
\hline Rhodus, 2006 [14] & $* *$ & $* *$ & $* *$ & 6 \\
\hline Sun, 2005 [15] & $* * *$ & $* *$ & $* *$ & 7 \\
\hline Sun, 2002 [16] & $* *$ & $*$ & $* *$ & 5 \\
\hline Liu, 2011 [17] & $* * * *$ & $* *$ & $* *$ & 8 \\
\hline Zhang, 2008 [18] & $* * *$ & $* *$ & $* *$ & 7 \\
\hline Rhodus, 2005 [19] & $* * * *$ & $* *$ & $* *$ & 8 \\
\hline Kaur, 2015 [20] & $* * * *$ & $*$ & $* *$ & 7 \\
\hline Yamamoto, 1994 [21] & $* * * *$ & $*$ & $* *$ & 7 \\
\hline
\end{tabular}

three months before entering the study. The mean serum IL-6 level in major type was significantly higher than that in minor type erosive OLP patients, suggesting that the serum IL-6 level may be associated with the severity of disease. Gu et al. [2] evaluated 20 OLP patients for IL-6 levels: 10 with erosive OLP, and 10 with reticular OLP. IL-6 level was significantly higher in both oral exfoliated cells and serum in patients with ulcerative OLP compared with patients with reticular OLP (two patients with ulcerative OLP were in a very active disease phase so their serum IL-6 levels were greater than $100 \mathrm{pg} / \mathrm{ml}$ and deleting the two cases did not change the trend). This could be due to local and systemic production of IL- 6 by many cell types. Therefore, the IL- 6 levels may change in types of OLP and with the severity of OLP.

Zhang et al. [18] demonstrated that there was a significant correlation between serum and salivary levels of
IL-6 in Chinese OLP patients. The levels of this cytokine in saliva went up with the levels of its serum partner in the OLP group. A study by Rhodus et al. [13] supported a significant increase level of IL-6 in patients with OLP and a positive correlation between increased serum and salivary IL-6 levels and clinical forms of OLP lesions [26]. Therefore, based on these results and meta-analysis, the saliva levels of IL-6 are greater than the serum levels, and when the saliva is more convenient and safer the evaluation of the levels of salivary IL-6 in OLP patients are proffered that the serum levels in future treatments.

\section{Limitations}

First, most studies for the evaluation of salivary IL-6 levels used small sample sizes. Second, the kits used for measurement of IL-6 levels had different sensitivities and 


\begin{tabular}{|c|c|c|c|c|c|c|c|c|}
\hline \multirow[t]{2}{*}{ Study name } & \multicolumn{3}{|c|}{ OLP group } & \multicolumn{3}{|c|}{ Control group } & \multirow[t]{2}{*}{ Std diff in means and $95 \% \mathrm{CI}$} & \multirow{2}{*}{$\begin{array}{l}\text { Relative } \\
\text { weight }\end{array}$} \\
\hline & Mean & $S D$ & $N$ & Mean & $S D$ & $\bar{N}$ & & \\
\hline Kaur, 2015 & 32.28 & 4.06 & 54 & 16.60 & 1.88 & 50 & & 17.62 \\
\hline Liu, 2011 & 3.64 & 4.47 & 21 & 7.91 & 4.05 & 43 & & 17.75 \\
\hline Rhodus, 2005a & 371.35 & 205.52 & 13 & 47.46 & 18.74 & 13 & $\mathbf{\square}$ & 17.46 \\
\hline Rhodus, 2005b & 148.12 & 21.30 & 13 & 2.26 & 0.72 & 13 & & 14.96 \\
\hline Rhodus, 2006 & 74.01 & 9.67 & 13 & 1.35 & 1.33 & 13 & & 14.56 \\
\hline Zhang, 2008 & 48.79 & 8.53 & 30 & 29.90 & 4.68 & 30 & 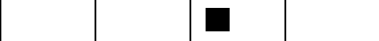 & 17.66 \\
\hline Total & & & 144 & & & 162 & & $100 \%$ \\
\hline $\begin{array}{l}\text { Heterogeneity: } \\
\text { Total for overa }\end{array}$ & m-effe & $Z=3$ & & ) & & & $\begin{array}{cccc}0 & -10.00 & 0.00 & 10.00 \\
\text { OLP } & & \text { Control }\end{array}$ & \\
\hline
\end{tabular}

Fig. 2. Forest plot of random-effect of interleukin-6 levels of saliva in OLP groups compared with healthy controls; Std diff - standard difference, OLP - oral lichen planus

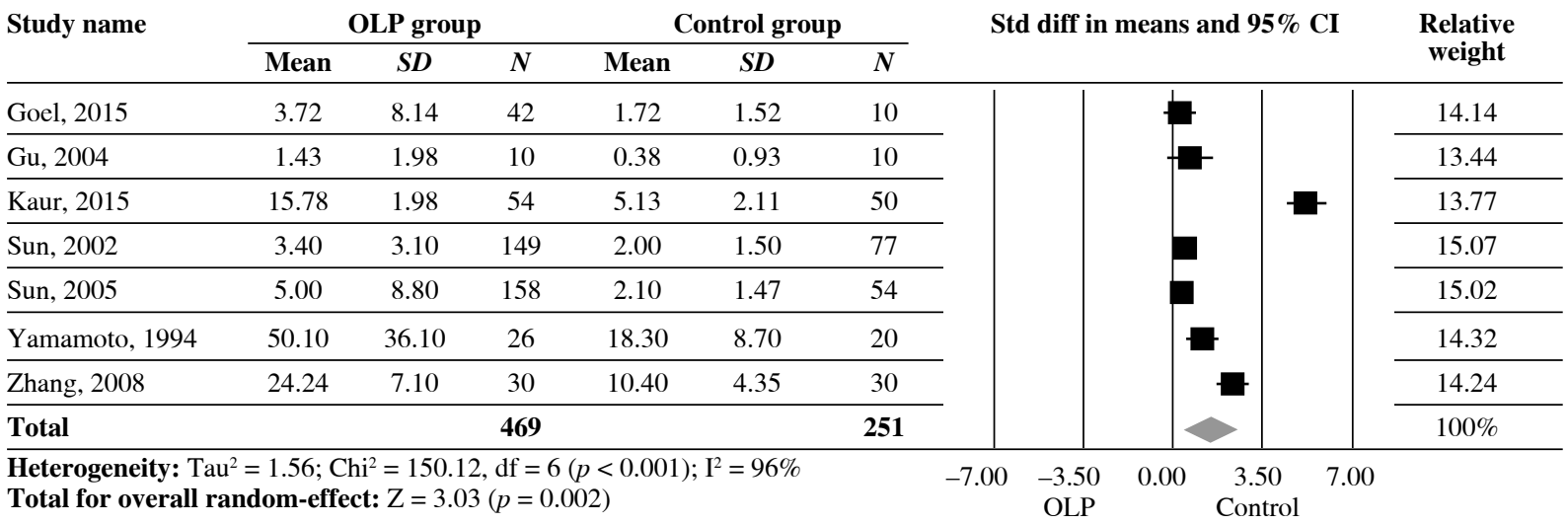

Fig. 3. Forest plot of random-effect of interleukin-6 levels of serum in OLP groups compared with healthy controls; Std diff - standard difference, OLP - oral lichen planus

A

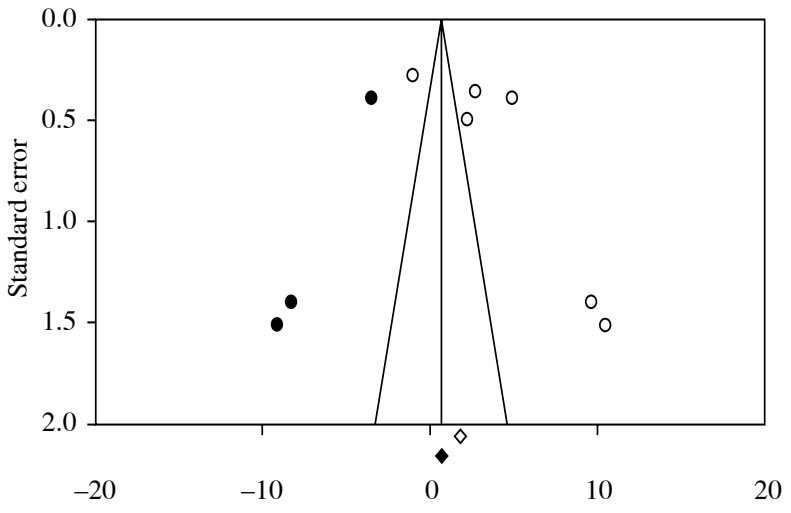

Std diff in means
B

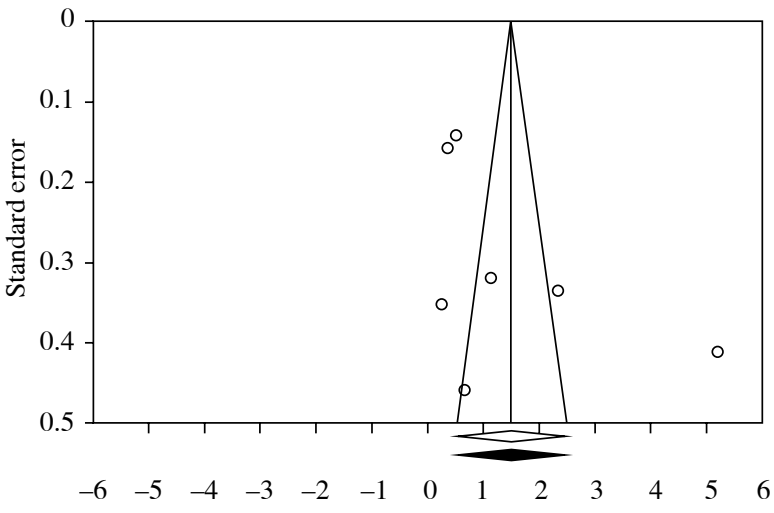

Std diff in means

Fig. 4. Funnel plot of random-effect of interleukin-6 of (A) saliva and (B) serum in oral lichen planus groups compared with healthy controls; Std diff - standard difference 
specificities. Third, in some studies, OLP patients and healthy controls were not age and sex matched. Fourth, the numbers of patients based on the type of OLP in metaanalyses were different. These limitations may create a high heterogeneity in the results of meta-analysis, although there was no publication bias between the studies, and these were of high quality.

\section{Conclusions}

This meta-analysis suggests that IL-6 may be an important biomarker, and it confirms the role of this cytokine in the pathogenesis of OLP. Also, the higher levels of IL-6 in saliva compared with serum suggest that measurement of this marker in saliva may be more useful than serum for diagnostic and therapeutic aims.

\section{Acknowledgements}

This work was supported by Kermanshah University of Medical Sciences.

The authors declare no conflict of interests.

\section{References}

1. Abdel-Haq A, Kusnierz-Cabala B, Darczuk D, et al. (2014): Interleukin-6 and neopterin levels in the serum and saliva of patients with Lichen planus and oral Lichen planus. J Oral Pathol Med 43: 734-739.

2. Gu GM, Martin MD, Darveau RP, et al. (2004): Oral and serum IL-6 levels in oral lichen planus patients. Oral Surg Oral Med Oral Pathol Oral Radiol Endod 98: 673-678.

3. Mozaffari HR, Rahmani M, Rezaei F, et al. (2016): Evaluation of oral lichen planus frequency in patients referred to pathology centers of Kermanshah city, during 2008 to 2011. Sch J App Med Sci 4: 2200-2202.

4. Sugerman PB, Savage NW, Walsh LJ, et al. (2002): The pathogenesis of oral lichen planus. Crit Rev Oral Biol Med 13: 350-365.

5. Goel S, Marwah A, Kaushik S, et al. (2015): Role of serum interleukin-6 in deciding therapy for multidrug resistant oral lichen planus. J Clin Exp Dent 7: e477-482.

6. Dreuw A, Hermanns HM, Heise R, et al. (2005): Interleukin-6-type cytokines upregulate expression of multidrug resistance-associated proteins in NHEK and dermal fibroblasts. J Invest Dermatol 124: 28-37.

7. Hibi M, Nakajima K, Hirano T (1996): IL-6 cytokine family and signal transduction: a model of the cytokine system. J Mol Med 74: 1-12.

8. Chang KP, Kao HK, Wu CC, et al. (2013): Pretreatment interleukin-6 serum levels are associated with patient survival for oral cavity squamous cell carcinoma. Otolaryngol Head Neck Surg 148: 786-791.

9. Tamura S, Ouchi KF, Mori K, et al. (1995): Involvement of human interleukin 6 in experimental cachexia induced by a human uterine cervical carcinoma xenograft. Clin Cancer Res 1: 1353-1358.
10. Hodge DR, Peng B, Cherry JC, et al. (2005): Interleukin 6 supports the maintenance of p53 tumor suppressor gene promoter methylation. Cancer Res 65: 4673-4682.

11. Nishimoto $N$ (2010): Interleukin-6 as a therapeutic target in candidate inflammatory diseases. Clin Pharmacol Ther 87: 483-487.

12. Jinno T, Kawano S, Maruse Y, et al. (2015): Increased expression of interleukin- 6 predicts poor response to chemoradiotherapy and unfavorable prognosis in oral squamous cell carcinoma. Oncol Rep 33: 2161-2168.

13. Rhodus NL, Cheng B, Myers S, et al. (2005a): A comparison of the pro-inflammatory, NF-kappaB-dependent cytokines: TNF-alpha, IL-1-alpha, IL-6, and IL-8 in different oral fluids from oral lichen planus patients. Clin Immunol 114: 278-283.

14. Rhodus NL, Cheng B, Bowles W, et al. (2006): Proinflammatory cytokine levels in saliva before and after treatment of (erosive) oral lichen planus with dexamethasone. Oral Dis 12: 112-116.

15. Sun A, Wang J, Chia J, et al. (2005): Serum interleukin-8 level is a more sensitive marker than serum interleukin-6 level in monitoring the disease activity of oral lichen planus. B J Dermatol 152: 1187-1192.

16. Sun A, Chia JS, Chang YF, et al. (2002): Serum interleukin-6 level is a useful marker in evaluating therapeutic effects of levamisole and Chinese medicinal herbs on patients with oral lichen planus. J Oral Pathol Med 31: 196-203.

17. Liu Y, Jin JQ, Yuan ZF, et al. (2011): [Levels of interlukin-6 and tumor necrosis factor- $\alpha$ in saliva of patients with type 2 diabetes mellitus and oral lichen planus]. Beijing Da Xue Xue Bao 43: 596-599.

18. Zhang Y, Lin M, Zhang S, et al. (2008): NF-kappaB-dependent cytokines in saliva and serum from patients with oral lichen planus: a study in an ethnic Chinese population. Cytokine 41: 144-149.

19. Rhodus NL, Cheng B, Myers S, et al. (2005b): The feasibility of monitoring NF-kappaB associated cytokines: TNF-alpha, IL-1alpha, IL-6, and IL-8 in whole saliva for the malignant transformation of oral lichen planus. Mol Carcinog 44: 77-82.

20. Kaur J, Jacobs R (2015): Proinflammatory cytokine levels in oral lichen planus, oral leukoplakia, and oral submucous fibrosis. J Korean Assoc Oral Maxillofac Surg 41: 171-175.

21. Yamamoto T, Yoneda K, Ueta E, et al. (1994): Serum cytokines, interleukin-2 receptor, and soluble intercellular adhesion molecule-1 in oral disorders. Oral Surg Oral Med Oral Pathol 78: 727-735.

22. Zheng Z, Zhang L, Zhu T, et al. (2016): Peripheral brain-derived neurotrophic factor in autism spectrum disorder: a systematic review and meta-analysis. Sci Rep 6: 31241.

23. Postal M, Appenzeller S (2011): The role of Tumor Necrosis Factor-alpha (TNF-alpha) in the pathogenesis of systemic lupus erythematosus. Cytokine 56: 537-543.

24. Eppel W, Worda C, Frigo P, et al. (2000): The influence of cotinine on interleukin 6 expression in smokers with cervical preneoplasia. Acta Obstet Gynecol Scand 79: 1105-1111.

25. Brailo V, Vucicevic-Boras V, Lukac J, et al. (2012): Salivary and serum interleukin 1 beta, interleukin 6 and tumor necrosis factor alpha in patients with leukoplakia and oral cancer. Med Oral Patol Oral Cir Bucal 17: e10-15.

26. Pezelj-Ribaric S, Prso IB, Abram M, et al. (2004): Salivary levels of tumor necrosis factor-alpha in oral lichen planus. Mediators Inflamm 13: 131-133. 